\title{
Atomic Resolution TEM Study on Quantum Dots in ZnSe/ZnTe Heterostructure
}

\author{
S. J. Kim*** W. Wang**, J. D. Phillips**, X. Q. Pan*
}

*Department of Materials Science and Engineering, University of Michigan, Ann Arbor, MI 48109

**Dept. of Electrical Engineering \& Computer Science, Univ. of Michigan, Ann Arbor, MI 48109

The intermediate band solar cell (IBSC) concept has recently attracted immense interest due to a high theoretical efficiency $63.1 \%$, surpassing the Shockley-Quiesser limit of $40.7 \%$ for a single energy-band solar cell [1]. In particular, metal chalcogenide quantum dots (QD), especially cadmium-based, have been so far recognized as a good candidate for possible applications in IBSCs. The high toxicity of cadmium UV illumination, though, makes it unsuitable for practical use in solar cells. $\mathrm{ZnTe}$ and $\mathrm{ZnSe}$ are the possible replacement for cadmium chalcogenides. However, narrow absorption spectra due to large energy band gaps limits device performance [2].

Type II band alignment, a staggered band offset structure, provides a potential barrier for one type of carrier (either electron or hole) and confines the other in QDs. This effectively separates charges and makes recombination spatially indirect, enhancing radiative life-times of charge carriers [3]. In this study, ZnSe buffer layers $(500 \mathrm{~nm})$ were deposited on p-type (100) GaAs substrate by reactive molecular beam epitaxy, followed by the multi-cycle growth of ZnTe QDs (3, 6, or 9 mono-layers per cycle) embedded in $\mathrm{ZnSe}$ layers $\left(20 \mathrm{~nm}\right.$ each) at $250^{\circ} \mathrm{C}$. A $50 \mathrm{~nm} \mathrm{ZnSe}$ capping layer was subsequently grown. Microstructure and defects of $\mathrm{ZnSe} / \mathrm{ZnTe} \mathrm{QD}$ heterostructure were then studied using atomic-resolution transmission electron microscopy (TEM) and aberration-corrected scanning TEM (STEM), as well as photoluminescence (PL) spectroscopy.

The typical microstructure of the $\mathrm{ZnSe} / \mathrm{ZnTe}$ QD heterostructure film on GaAs substrate is shown in Fig. 1. The ZnTe wetting layers in which the QDs are embedded is not straight, most likely due to the surface energy of ZnTe counterbalancing with strain energies. STEM images of wetting layers within a rough film surface shows the irregular arrangement of quantum dots of various sizes (Fig. 2(a)), with their edges causing enough strain around to form stacking faults (Fig. 2(b)). The presence of quantum confinement is confirmed by a PL signal at $680 \mathrm{~nm}$. The dominant type of defects in the ZnSe bulk is extrinsic stacking faults bounded by the two Shockley partials with the Burgers' vector $\boldsymbol{b}=\boldsymbol{a} / 2<112>$. Defects often form complex networks by linking one another in Z-shaped formations (Fig. 3(a)). High density of stacking faults was identified near ZnTe/ZnSe interfaces; these stacking faults start from and terminate at the interfaces and often form in symmetry (Fig. 3(b)).

In summary, our studies have shown that a type-II $\mathrm{ZnTe} / \mathrm{ZnSe}$ QD device has wide tunable absorption spectrum, low crystallization temperature, increasing efficiency while reducing the cost. Atomic-level studies of $\mathrm{ZnTe} / \mathrm{ZnSe}$ interfacial structures contribute not only to optimizing growth conditions but also to understanding fundamental device physics for expanded applications.

[1] A. Luque et al., Phys. Rev. Letts. 78 (1997) 5014-5017

[2] J. Bang et al, Chem. Mater. 22 (2010) 233-240

[3] S. Kim et al, J. Am. Chem. Soc. 125 (2003) 11466-11467

[4] This work was supported through The University Michigan Energy Frontier Research Center funded by the DOE/BES under award number DE-SC0000957. 


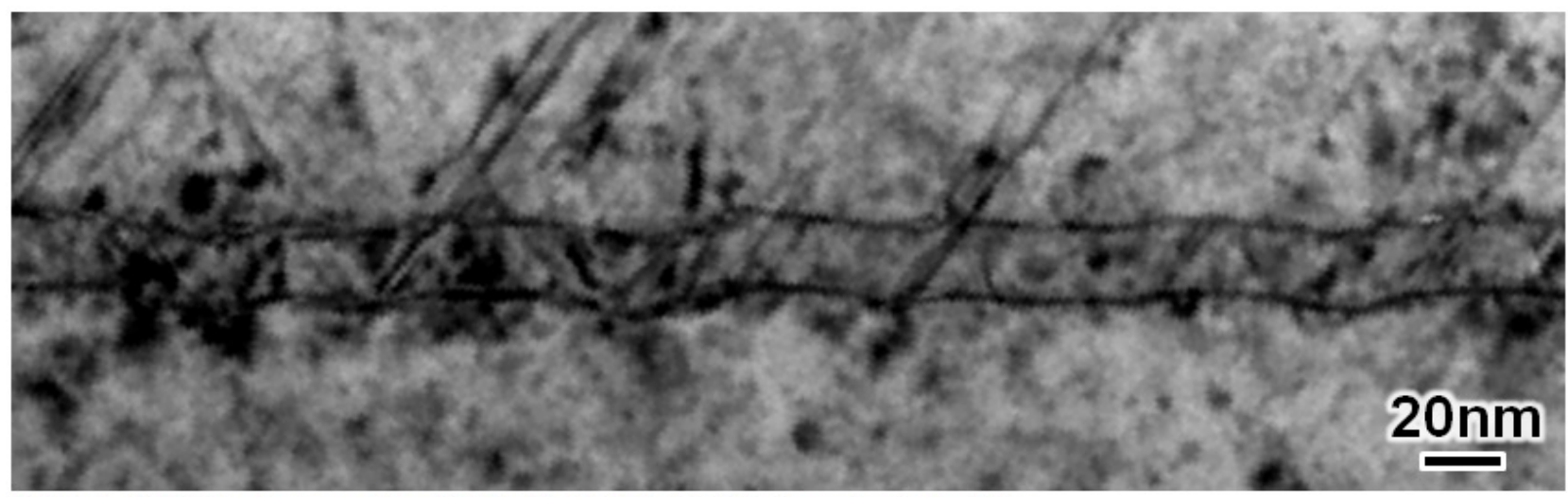

FIG. 1. TEM micrograph of a $\mathrm{ZnSe} / \mathrm{ZnTe} \mathrm{QD}$ heterostucture film

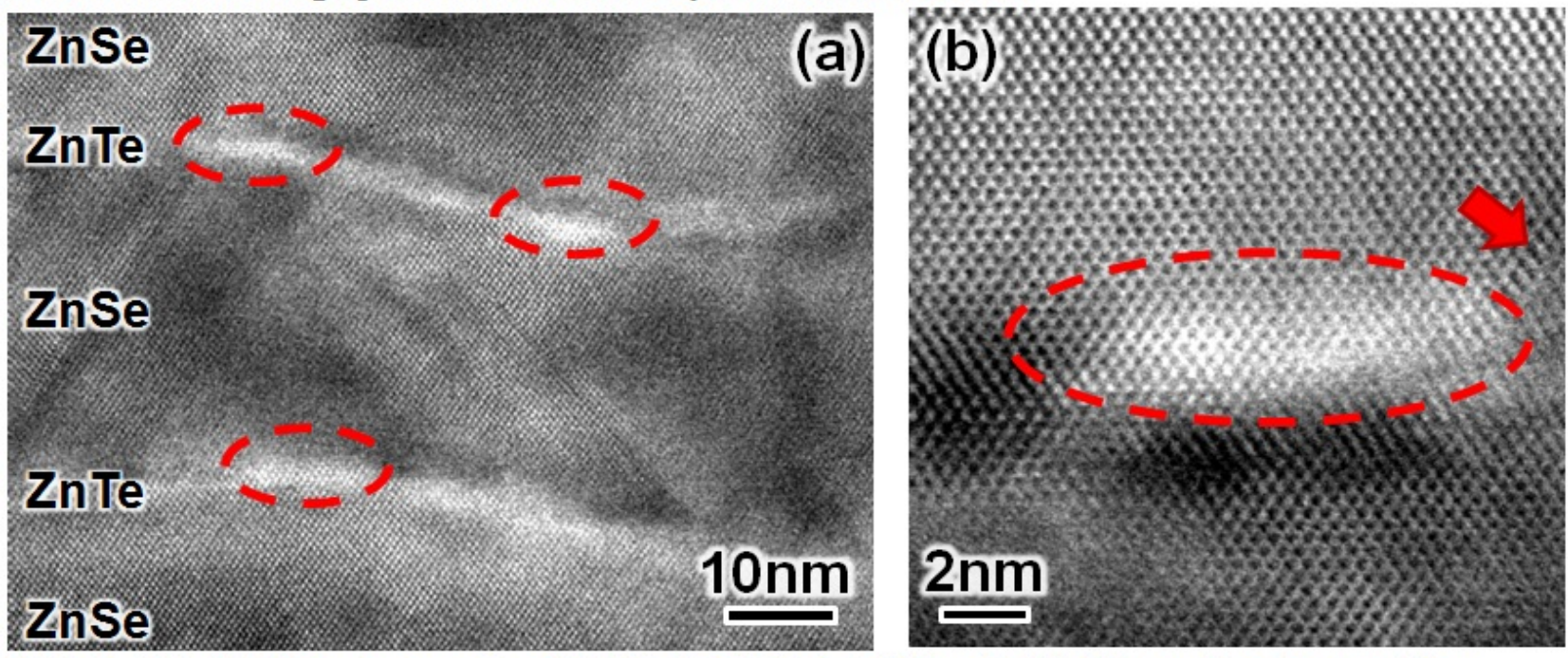

FIG. 2. HR-STEM micrographs of (a) ZnTe QD's (circle) embedded in wetting layers, (b) a single ZnTe QD (circle) next to a stacking fault (arrow)

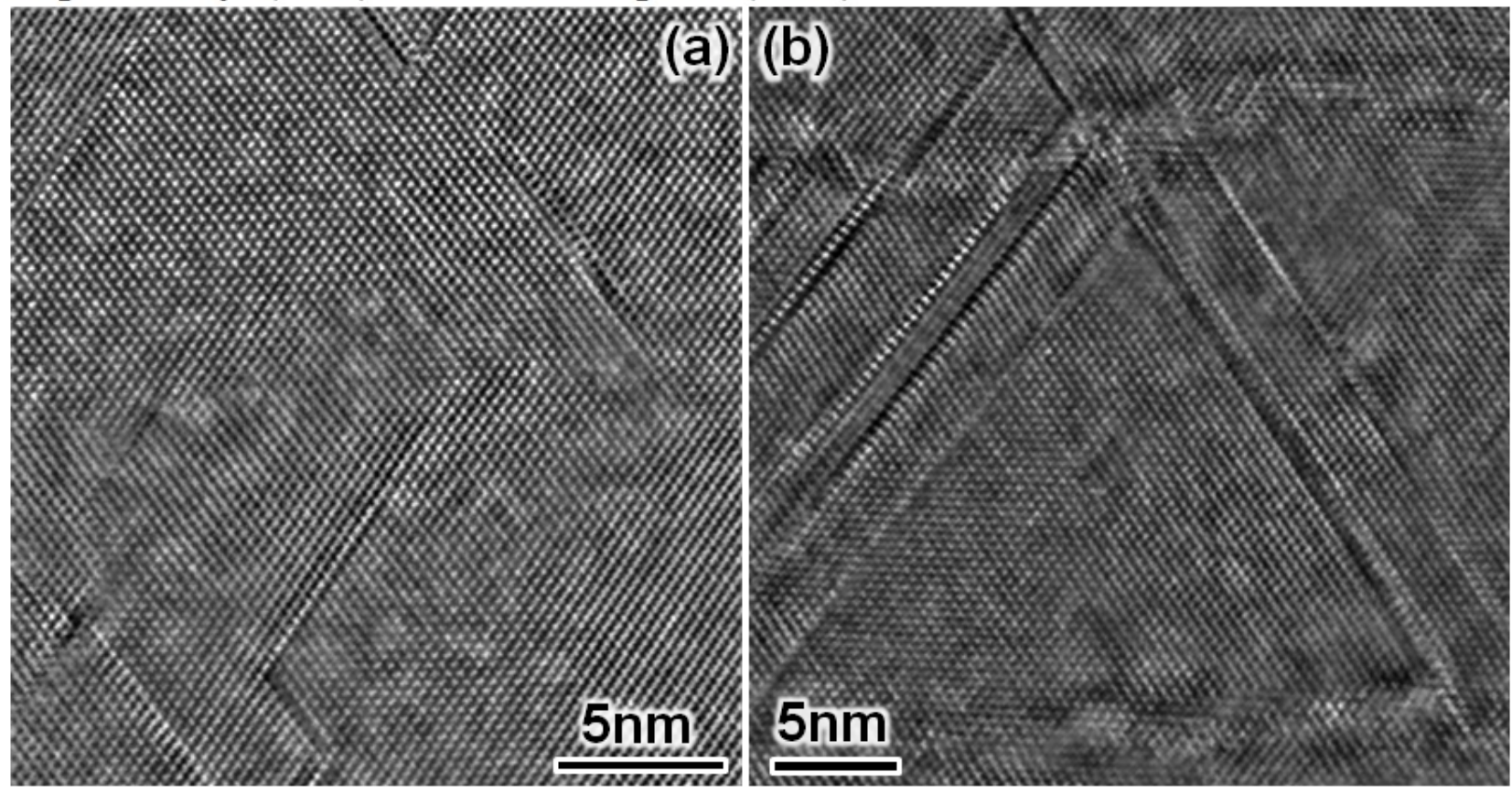

FIG. 3. TEM images of (a) extrinsic stacking fault bounded by Shockley partials and (b) stacking faults forming symmetry at the wetting layer in $\mathrm{ZnSe} / \mathrm{ZnTe} \mathrm{QD}$ heterostucture film 